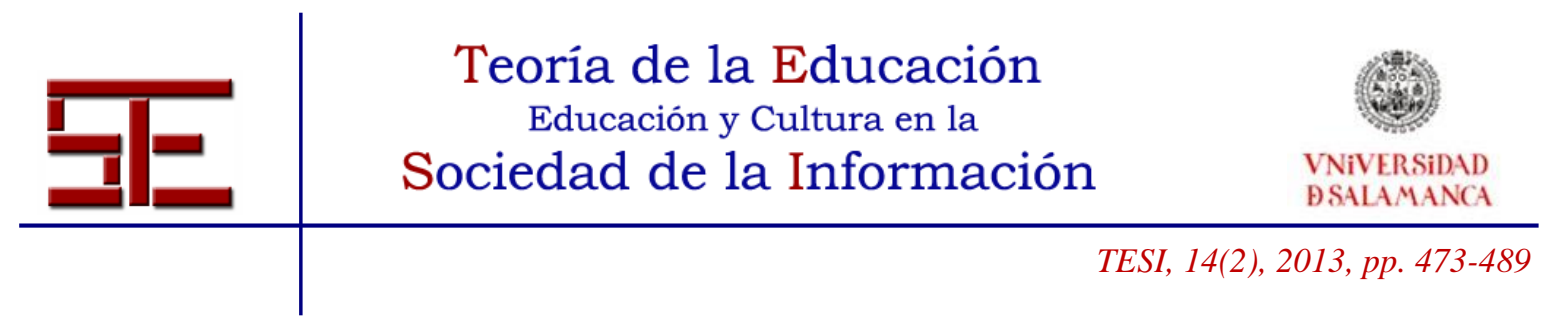

\title{
EVALUACIÓN DE LA CALIDAD DE LOS PROCESOS DEL E-LEARNING: UNA PROPUESTA CON NUEVAS DIMENSIONES
}

Resumen: La era de la globalización en la educación aumenta la competitividad de las instituciones de enseñanza, especialmente en la educación superior. El método para la diferenciación de las instituciones es el factor de calidad. Con relación a la calidad en la modalidad de e-learning, pueden encontrarse discusiones interesantes sobre la complejidad de la adopción de una metodología. Por lo tanto, este artículo tiene como objetivo presentar el estado del arte de la calidad de los procesos e-learning y una propuesta de nuevas dimensiones a tener en cuenta al evaluar la calidad de los procesos e-learning.

Palabras clave: calidad del e-learning; educación a distancia; e-learning.

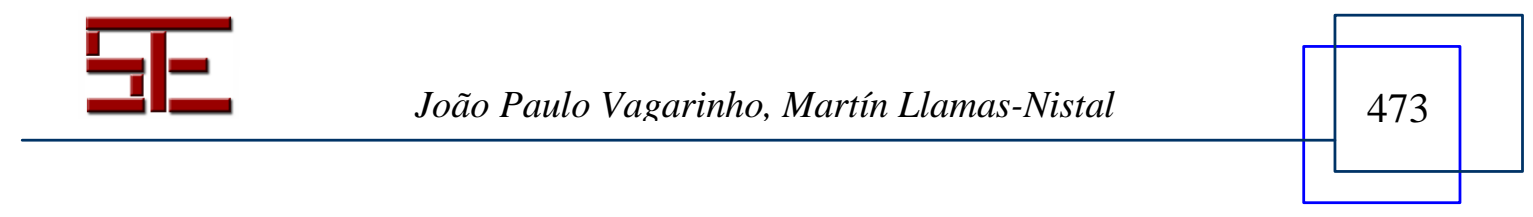




\section{AVALIAÇÃO NA QUALIDADE DOS PROCESSOS DO E-LEARNING: UMA PROPOSTA COM NOVAS DIMENSÕES}

Resumo: A era da globalização na educação aumentou a competitividade entre as instituições de ensino especialmente no ensino superior. $\mathrm{O}$ método para a diferenciação das instituições é o factor qualidade. Na modalidade de ensino e-learning associado ao tema qualidade podemos encontrar debates interessantes sobre a complexidade de adoptar uma metodologia. Desta forma este artigo tem como finalidade apresentar o estado de arte da qualidade orientada aos processos no e-learning e uma proposta para as novas dimensões a ter em conta na avaliação da qualidade do e-learning orientada aos processos.

Palavras chave: qualidade do e-learning; ensino à distância; e-learning. 


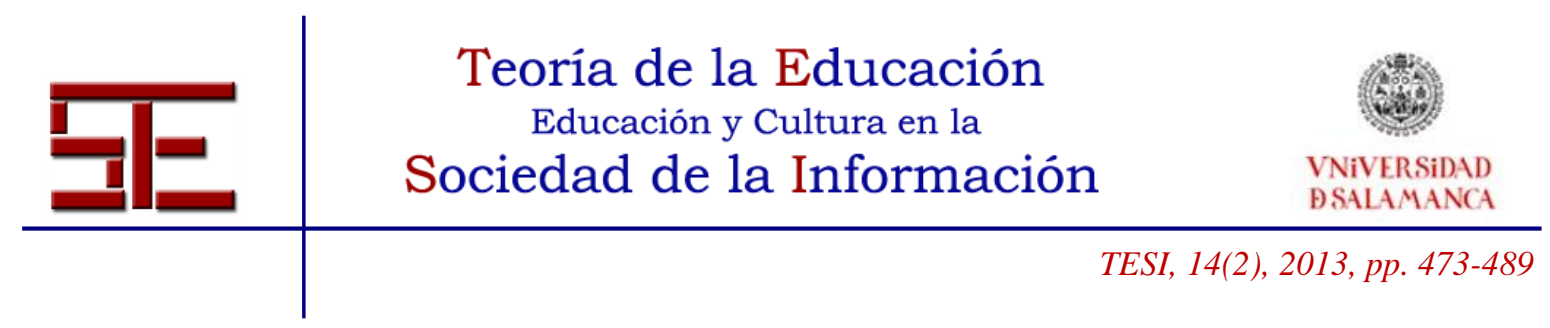

\title{
ASSESSMENT OF THE QUALITY IN E-LEARNING PROCESSES: A PROPOSAL WITH NEW DIMENSIONS
}

\begin{abstract}
The globalization in education increased competitiveness among institutions of learning especially in higher education. The method for the differentiation of institutions is the quality factor. In e-learning quality we can find interesting discussions about the complexity of adopting a methodology. This article show the state of art quality oriented processes in e-learning and a proposal for new dimensions to be considered in assessing the quality of e-learning-oriented processes.
\end{abstract}

Key Words: quality in e-learning; distance learning; e-learning.

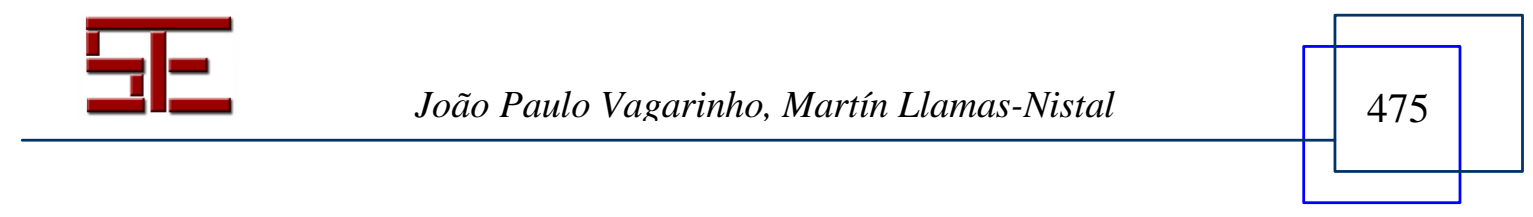




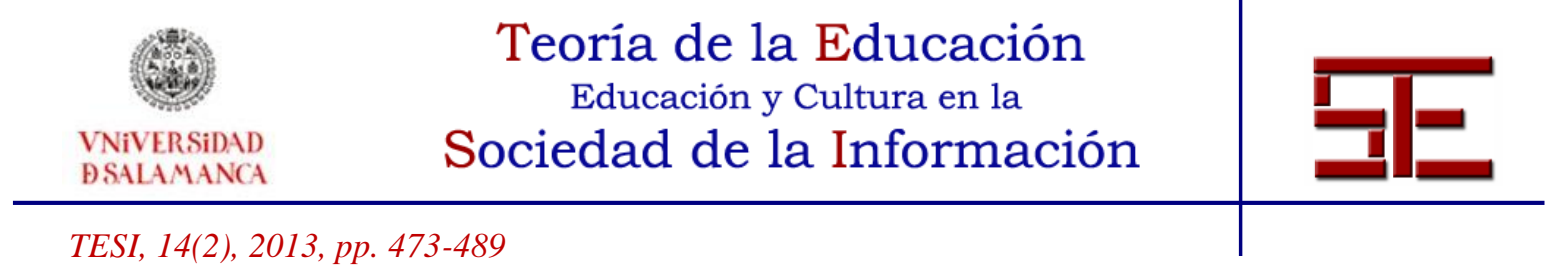

\section{AVALIAÇÃO NA QUALIDADE DOS PROCESSOS DO E-LEARNING: UMA PROPOSTA COM NOVAS DIMENSÕES}

Fecha de recepción: 18/01/2013; fecha de aceptación: 01/04/2013; fecha de publicación: 30/07/2013

João Paulo Vagarinho

joao.vagarinho@gmail.com

Universidade de Vigo

Martín Llamas-Nistal

martin@gist.uvigo.es

Universidade de Vigo

\section{1.- INTRODUÇÃO.}

A aceleração no desenvolvimento das telecomunicações e o grande crescimento na utilização da Internet possibilitou enormes e variadas transformações nas organizações de ensino e principalmente na forma de interagir dos intervenientes (organizações, professores e alunos). Com esta forma de interacção o ensino à distância evoluiu para uma nova geração (Meirinho y Osório 2006, 27-28).

Assim, das várias modalidades do ensino à distância o e-learning surge em destaque, este é definido (Cardoso, 2007, 7) com base em Masie (1999) como "É o uso de tecnologia de rede para desenhar, produzir, seleccionar, gerir e ampliar a aprendizagem”.

O Processo de Bolonha assinado em 1999 por vários Ministros Europeus teve como ponto fundamental, executar acções conjuntas relativas ao ensino superior em todos os Países da Comunidade Europeia para elevar a competitividade em relação às outras zonas geográficas do mundo. Para atingir esta meta foram traçados objectivos dos quais se destaca "Fomentar a cooperação entre os Países da Comunidade Europeia para avaliar e garantir a qualidade através de métodos e critérios comparáveis" (EACEA 2009, 13-18) e (DGES 2010, sem paginação). Em 2003 na cidade de Berlim os Ministros reforçam um aperfeiçoamento no sistema de garantia de qualidade no ensino superior a nível institucional, Nacional e Europeu.

De acordo com (Gomes 2006, 35-38) e tendo por base um documento regulador de instrumentos, é reconhecido que existe necessidade de "adaptar o processo de

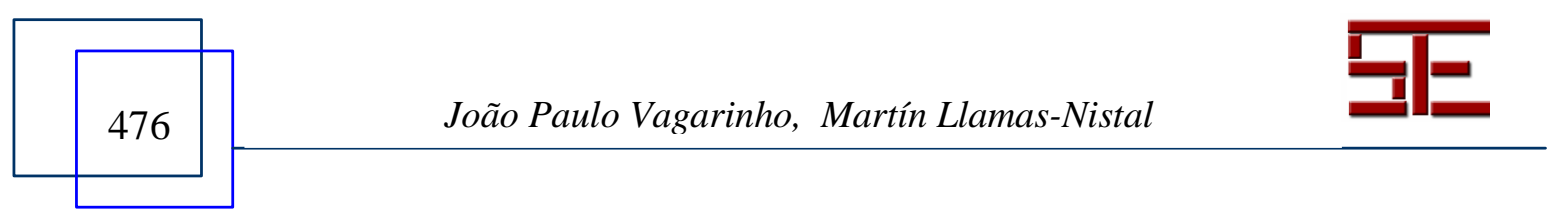




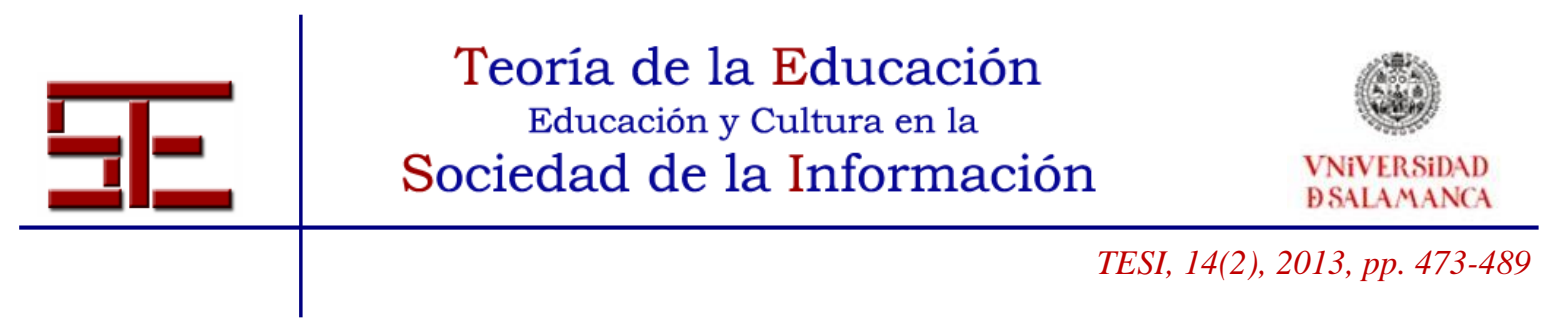

aprendizagem aos conceitos e perspectivas da sociedade moderna e aos meios tecnológicos disponíveis" e neste domínio o e-learning é uma possibilidade forte pois contribui directamente para três linhas principais do Processo de Bolonha: Promover a dimensão europeia do ensino superior; promover a aprendizagem ao longo da vida e promover a mobilidade.

De acordo com o exposto o presente artigo inicia com uma definição sobre a qualidade no e-learning, de seguida faz uma abordagem sobre os standards existentes e as categorias em que se dividem. Posteriormente descrevemos a avaliação da qualidade do e-learning orientado aos processos, os níveis existentes e a selecção de entidades para o trabalho realizado.

Finalmente analisamos os itens e categorias de cada entidade e ficaremos a conhecer o estado de arte da qualidade orientada aos processos no e-learning, com base no estado de arte "nasce" uma proposta para as novas dimensões a ter em conta na avaliação da qualidade do e-learning orientada aos processos.

\section{2.- QUALIDADE NO E-LEARNING.}

A ideia da qualidade na educação é relativamente recente, em 1998 apenas seis países europeus tinham alguma forma de controlo de qualidade, actualmente existem mais de 200 organizações mencionadas na INQAAHE (Montalvo, Luna y Montenegro 2010, 1011). É difícil encontrar um consenso para a definição de qualidade uma vez que este conceito não é unitário mas sim aberto a várias perspectivas e dimensões, ainda assim, (Montalvo, Luna y Montenegro 2010, 10-11) recorrendo a uma citação de Pawlowski (2007) refere-se à qualidade do e-learning como "Cumplimiento adecuado de los objetivos y 472 ecnologias 472 de las personas involucradas, como resultado de un 472 ecnolog de negociación transparente y participativa dentro de una organización. Además, en el ámbito del e-learning, la calidad está relacionada com los 472ecnologi, 472ecnolog y servicios del aprendizaje, la educación y la formación, que están soportados por el uso de la información y las 472ecnologias de la comunicación".

\section{3.- UMA PEQUENA ABORDAGEM AOS STANDARDS.}

Uma das questões a debater na área do e-learning é o problema de normalização. Muitos dos problemas encontrados são por falta de standards contudo existem actualmente várias instituições a nível mundial a trabalhar em especificações para a criação de normas que

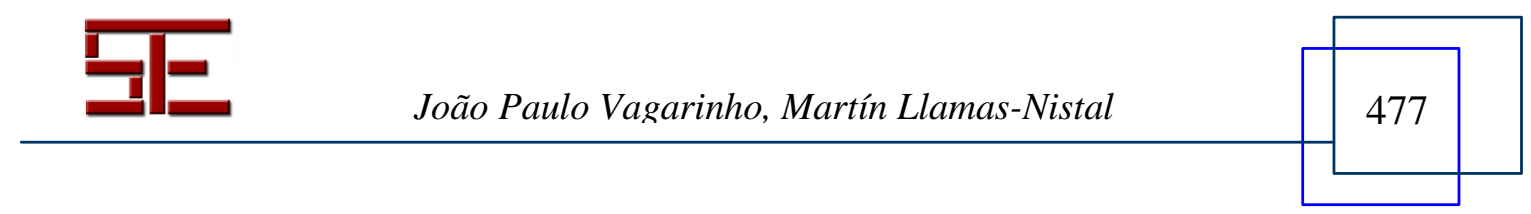




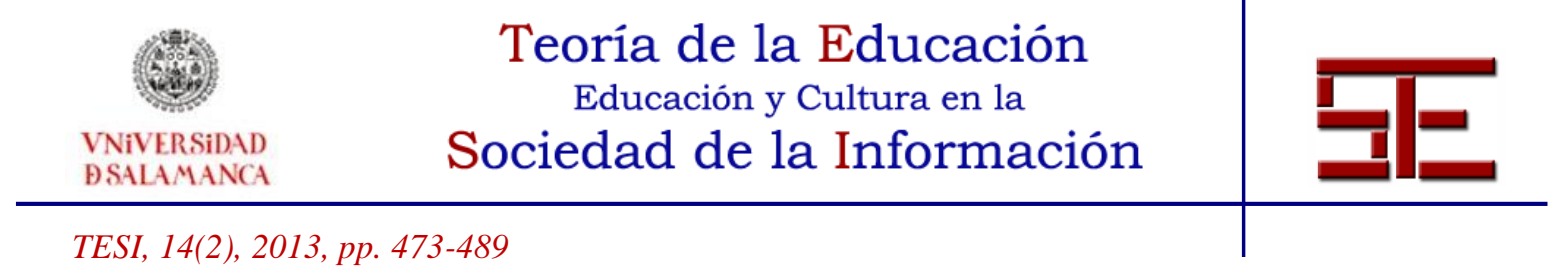

visam incentivar o uso de e-learning com todas as suas boas práticas, [7]. De uma forma geral, um standard é um processo que tem como objectivo auxiliar uma ou mais referência(s)/modelo(s) para empresas ou instituições onde estão definidas regras/boas práticas comuns e sem prejudicar a capacidade competitiva, pretende-se portanto compatibilidade e interoperabilidade, (Manjón et al 2007, 10).

Hoje em dia de acordo com (Ehlers y Pawlowski 2006, 24-25) e (Montalvo, Luna y Montenegro 2010, 10-11) os standards relativos ao e-learning estão divididas em três grandes grupos (figura 1):

\section{1.- Standards da Gestão e garantia de qualidade}

Apoiam o desenvolvimento da qualidade de acordo com as necessidades e exigências concretas das organizações. Permitem melhorar a flexibilidade, a reutilização, a transparência e comparabilidade. Normalmente são utilizados para processos, produtos e competências (área que nos interessa para este trabalho).

\section{2.- Standards da tecnologia de aprendizagem}

Fazem a interoperabilidade dos componentes dos ambientes de aprendizagem como por exemplo: os sistemas de autoria, os LMS, recursos de aprendizagem e serviços. São constituídas por normas de conteúdo, gestão, atores e didáctica. Contribuem para o cumprimento dos objectivos de qualidade da interoperabilidade.

\section{3.- Standards da tecnologia de internet}

São desenvolvidos para outros domínios, isto é, são utilizados (com normas de qualidade ou normas tecnológicas de aprendizagem) como tecnologia ou normas legais. 

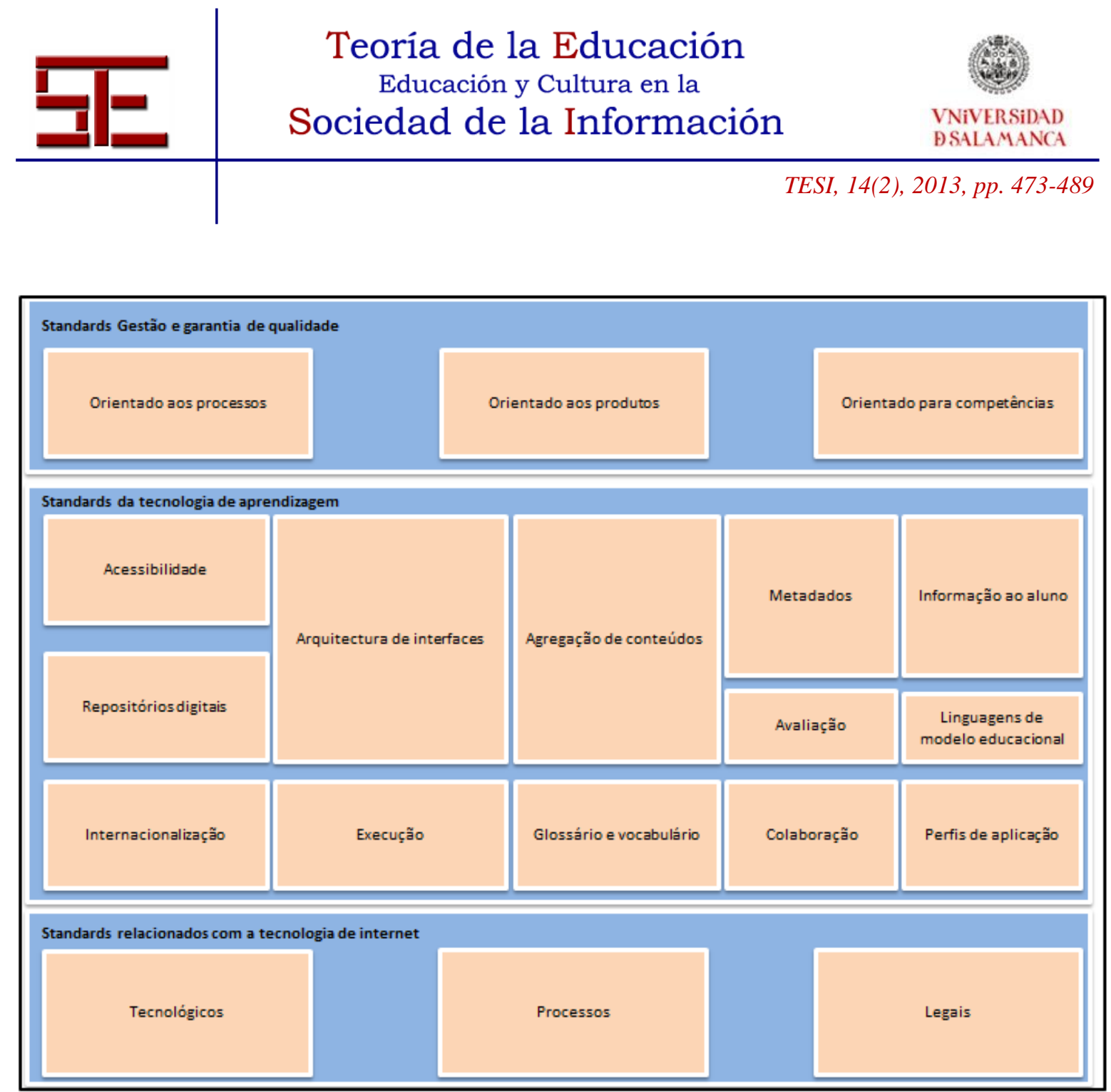

Figura 1: Classificação dos standards do e-learning, adaptado de Montalvo, Luna y Montenegro (2010).

\section{4.- AVALIAÇÃO DA QUALIDADE DO E-LEARNING ORIENTADO AOS PROCESSOS.}

Para este trabalho interessa-nos reflectir sobre a avaliação da qualidade do e-learning orientada aos processos e este grupo divide-se em três grandes categorias (Dondi y Moretti 2006, 24-25):

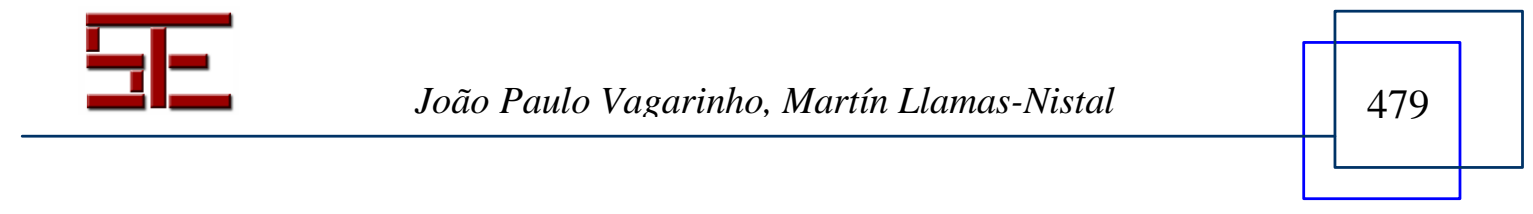




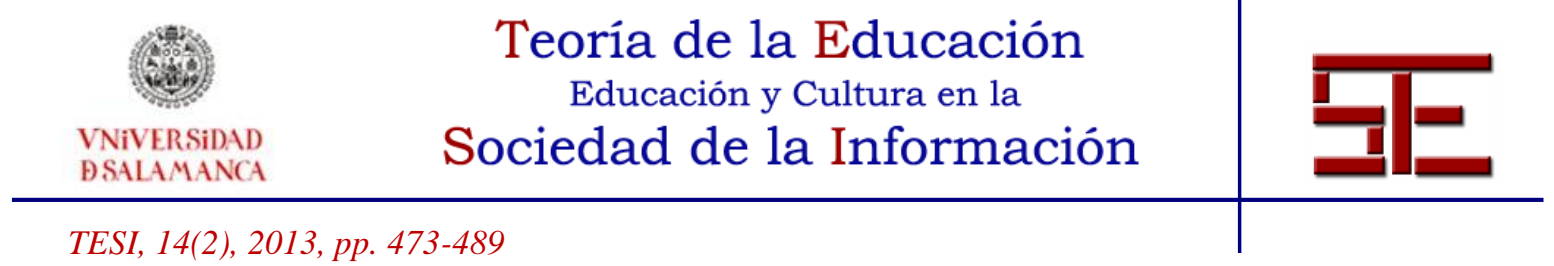

\section{1.- Qualidade nos processos gerais}

Neste grupo encontramos standards e boas práticas que não são específicas de um sector de actividade, logo podem ser adaptadas ao e-learning (por exemplo TQM, EFQM, Malcolm Baldrige, Demmin, entre outros).

\section{2.- Qualidade nos processos específicos}

Este grupo contém os standards e boas práticas que são específicas do e-learning, é um grupo que nos interessa analisar para este trabalho e por esta razão seleccionámos seis exemplos de entidades Europeias e Americanas. Algumas pesquisas de rankings das melhores Universidades de Ensino à Distância do mundo, mostram que as Universidades dos Estados Unidos da América e do Reino Unido estão no top desses rankings. Por outro lado, no Reino Unido nasceu a Open University (a primeira Universidade baseada no conceito total de educação à distância), desta forma, no Reino Unido seleccionámos a QAA e a ODLQC. Para além das instituições do Reino Unido também foi seleccionado o modelo da Noruega, este país é reconhecido por utilizar métodos e tecnologias inovadoras, desenvolveu à alguns anos dois modelos que despertaram interesse internacional: o modelo em rede e o modelo de educação combinada e assim, seleccionámos a NADE. Quanto às instituições Americanas, possuem uma enorme variedade de Universidades nesta modalidade de ensino e conseguiram criar e adaptar standards e boas práticas, logo podem dar um contributo válido para a Europa, no sentido de melhorar a avaliação da qualidade no e-learning orientada aos processos, neste caso seleccionámos a C-RAC, AFT e IHEP. Pelo exposto, as entidades a estudar são:

\subsection{1.- Quality Assurance Agency for Higher Education (QAA)}

É uma agência de certificação da qualidade e tem como missão melhorar a qualidade no ensino superior do Reino Unido, ou seja, fornecer informação, publicar relatórios e apoiar (aconselhar e orientar) as organizações de ensino nas boas práticas neste sentido desenvolveu e publicou um código de boas práticas, esse código é constituído por três partes. A parte de boas práticas que faz referência à garantia de qualidade no ensino à distância divide-se em quatro dimensões, essas dimensões possuem critérios (Dondi y Moretti 2006, 24-25) e (Miranda y Teixeira 2003, 122) relativos aos seguintes temas: alunos (aprendizagem), avaliação (aprendizagem formal e feedback dos alunos), curso (ambientação, aprovação, garantia de qualidade, gestão e monitorização), informação

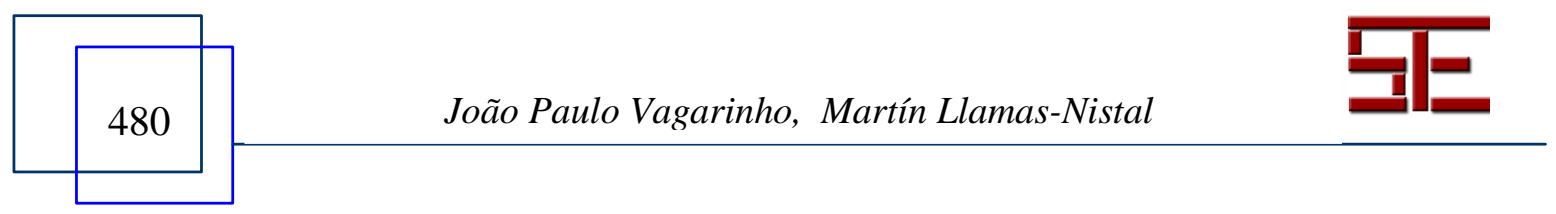




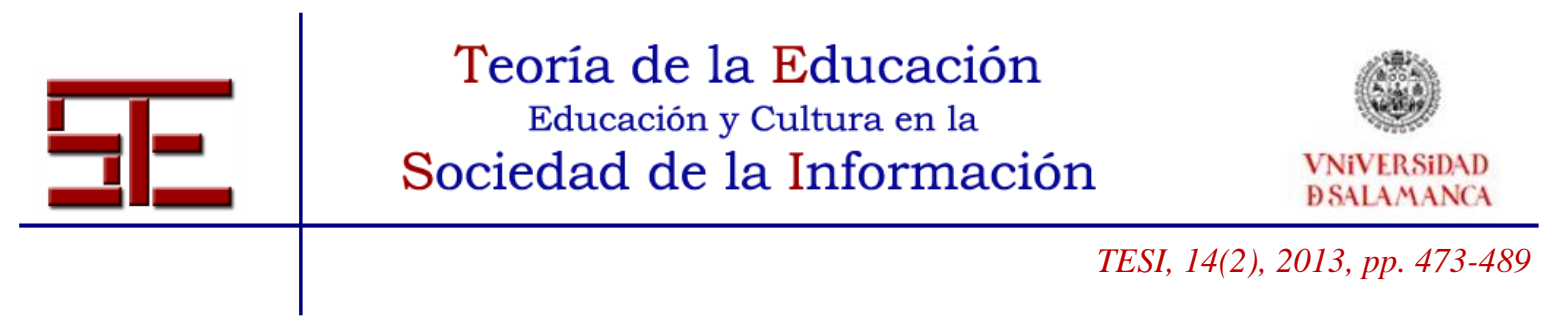

(cursos, eventos e representação dos alunos), institucional (estratégia e objectivos), leis e recursos (finanças);

\subsection{2.- Open \& Distance Learning Quality Council (ODLQC)}

É uma organização no Reino Unido fundada a pedido do governo, tem como missão ser um conselho de Qualidade no EaD. Possui como objectivo principal, ser uma referência, produzir orientações, identificar e melhorar a qualidade na educação, para tal criou um conjunto de normas para o EaD e todas as organizações que o aplicarem podem solicitar a acreditação. Os padrões da ODLQC para a garantia de qualidade no EaD na sua última versão em 2005 descrevem os temas (ODLQC 2005, 1-4): alunos (suporte à aprendizagem e deveres), avaliação (aprendizagem formal), cultura de qualidade (melhoria continua), curso (aprovação, estrutura, promoção e monitorização), ética (institucional), informação (cursos e avaliações), institucional (prestação de serviços, missão e visão), leis, pedagogia (modelo pedagógico, objectivos e recursos de aprendizagem), recursos (finanças e RH), segurança (informação) e suporte (institucional, orientação, assessoria e aconselhamento);

\subsection{3.- Norwegian Association for Distance Education (NADE)}

O primeiro país a criar leis para controlar a qualidade das organizações privadas de ensino à distância foi a Noruega em 1948. Em 1992 o Ministério de Educação da Noruega criou a NADE para rever a legislação do sistema de controlo criado em 1948. Assim, a NADE desenvolveu padrões de qualidade no ensino à distância (Rekkedal 2006, 1-13), esses padrões têm por base uma matriz (com 16 elementos) composta por áreas, em que cada área corresponde a um problema possível de avaliar (alunos, professores, cursos e a organização) nomeadamente em termos de condições e restrições, processos e resultados. Para este trabalho e de acordo com a matriz da NADE é interessante verificar quais os temas abordados no item "Organização", estes são: avaliação (organização), cultura de qualidade (melhoria continua e revisão periódica), informação (financeira, auditores e estrutura), institucional (objectivos), leis e recursos (finanças e formação);

\subsection{4.- Council of Regional Accrediting Commissions (C-RAC)}

É uma instituição das seis comissões regionais dos Estados Unidos que credenciam a concessão de instituições. O C-RAC propõe um guia de recomendações de qualidade composto por 29 itens agrupados em cinco componentes independentes e ligados a uma

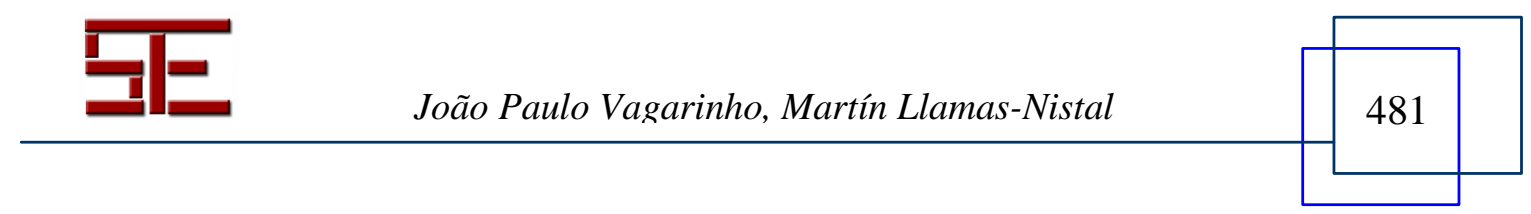




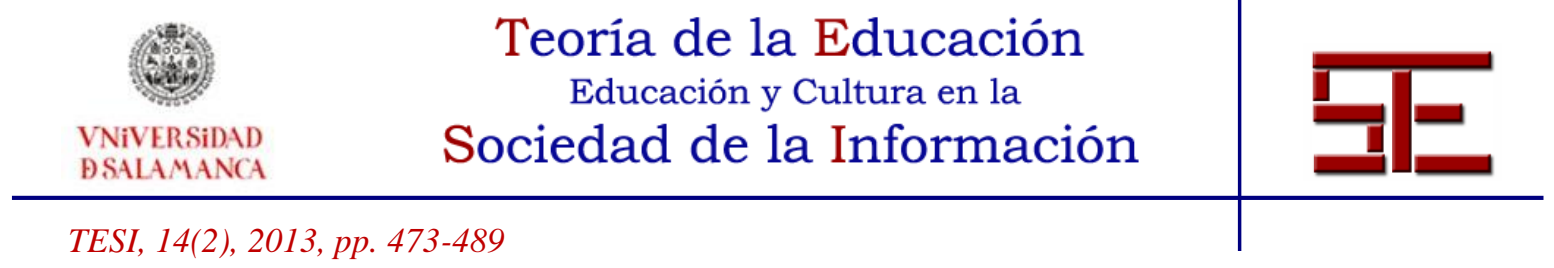

área específica, os componentes descrevem os seguintes temas (WCET 2001, sem paginação): acreditação (requisitos), alunos (suporte à aprendizagem e requisitos de admissão), avaliação (aprendizagem formal, potenciais projectos e cursos), cultura de qualidade (melhoria continua), curso (desenvolvimento, eficácia, estrutura, garantia de qualidade e gestão), ética (questões legais), informação (cursos, declarações politicas, pagamentos), institucional (contratos, marketing, politica de transferências, missão e visão), leis, pedagogia (manual de acolhimento, meio, objectivos, recursos de aprendizagem e supervisão), professores (competências e habilidades), recursos (finanças, formação, RH e instalações), segurança (informação e mecanismos de controlo) e suporte (administrativo, alunos, infra-estrutura, institucional, professores, programas, serviços, técnico, orientação, assessoria e aconselhamento);

\subsection{5.- Institute for Higher Education Policy (IHEP)}

É uma organização dos Estados Unidos da América que se dedica a promover o sucesso e acesso no ensino superior de acordo com as políticas públicas, orientadas para encontrar respostas aos grandes desafios do País em matéria de educação. Em Abril de 2000, o IHEP realizou um estudo onde identificou padrões de referência essenciais para garantir a excelência da qualidade em cursos superiores à distância utilizando a Internet e o resultado desse estudo foi a identificação de 24 padrões agrupados por 7 categorias (IHEP 2000, 9-10): alunos (suporte à aprendizagem), avaliação (organização), cultura de qualidade, curso (melhoria continua), informação (segurança) e suporte (professores e técnico);

\subsection{6.- American Federation of Teachers (AFT)}

É um sindicato que representa professores e outros profissionais da educação, tem como objectivo dar voz aos seus profissionais, fortalecer as instituições para onde trabalham, melhorar a sua qualidade de serviços, promover a democracia, direitos humanos e ajudar os membros. Na divisão da Educação Superior a AFT tem trabalhado activamente sobre temas relacionados com a $\mathrm{EaD}$ e a tecnologia principalmente incentivando os seus membros a terem um papel activo nas tecnologias educacionais, nas organizações de ensino. No ano 2000 a AFT, tendo por base um inquérito, criou um guia de boas práticas com catorze normas onde os temas são (AFT 2000, 7-15): acreditação (requisitos), avaliação (aprendizagem formal e organização), curso (aprovação, desenvolvimento, gestão e transferências), informação (cursos), materiais (reutilização), pedagogia (meio, modelo pedagógico e recursos de aprendizagem), professores (competências e

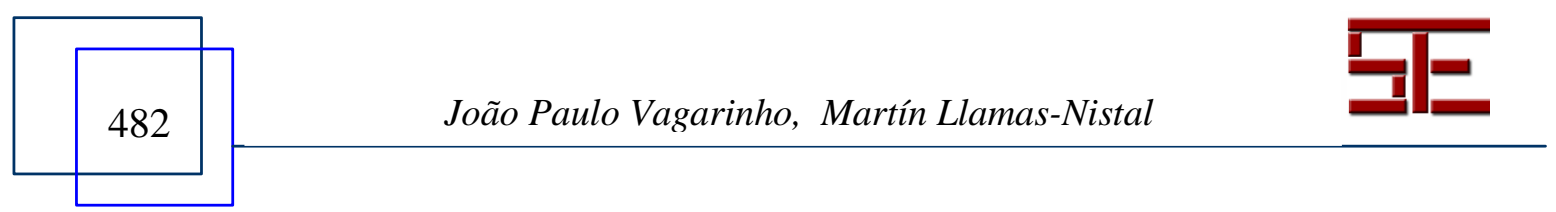




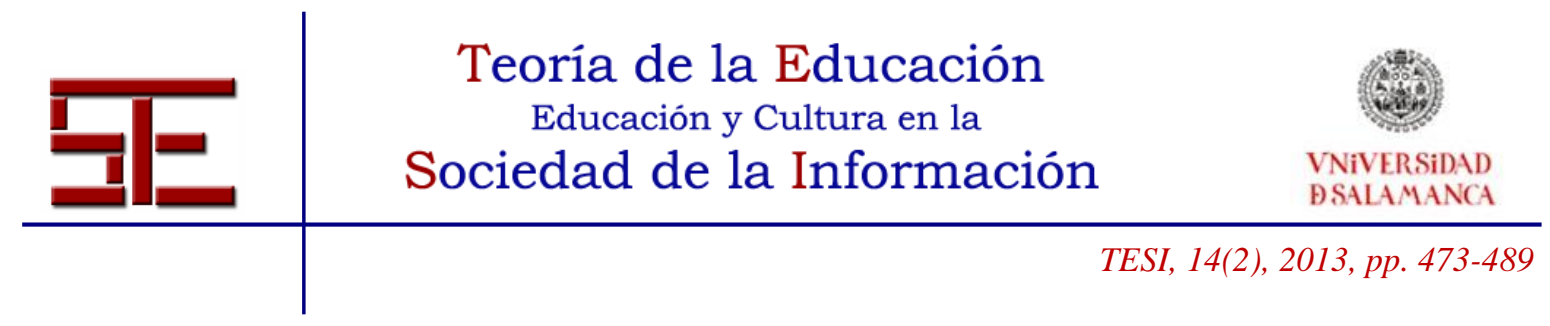

habilidades), recursos (formação e RH), segurança (mecanismos de controlo) e suporte (alunos e serviços).

\section{3.- Projectos na Europa para a qualidade do e-learning}

Neste grupo encontramos projectos de instituições que tentam promover os standards e boas práticas que são específicas do e-learning.

\section{5.- PEQUENA ANALISE.}

Como foi apresentado anteriormente todas as entidades defendem um conjunto de boas práticas, que de acordo com o seu ponto de vista, entendem que as mesmas são as que melhor se enquadram para avaliar a qualidade. Se juntarmos todos os standards apresentados pelas entidades aqui estudadas e agrupar esses mesmos standards, verificamos que nenhuma entidade consegue cobrir todos os temas (itens da tabela 1) e muito menos as categorias desses itens. Assim, a tabela 1 mostra a distribuição dos vários itens e respectivas categorias por cada entidade. Podemos observar que a entidade " $\mathrm{C}$ RAC" é aquela que consegue englobar mais itens mas não faz referência aos "Materiais". Por outro lado, neste contexto, esta tabela mostra bem a falta de cobertura dos standards de cada entidade relativamente aos itens apresentados. Mas cada item possui o seu nível de detalhe (categorias) e nesta perspectiva verificamos que as entidades estão longe de englobar todos os itens e categorias mencionados, mesmo aqueles que nos davam a entender englobar todo o item, na verdade isso não acontece.

\begin{tabular}{|c|c|c|c|c|c|c|c|}
\hline ITEM & CATEGORIA & QAA & NADE & IHEP & C-RAC & AFT & ODLQC \\
\hline \multicolumn{2}{|c|}{ 1. Acreditação (Requisitos) } & & & & $\mathrm{X}$ & $\mathrm{X}$ & \\
\hline \multicolumn{8}{|c|}{ 2. Alunos } \\
\hline & 2.1 Aprendizagem - Estratégia & $\mathrm{X}$ & & & & & \\
\hline & 2.2 Aprendizagem - Sucesso & $\mathrm{X}$ & & & $\mathrm{X}$ & & $\mathrm{X}$ \\
\hline & 2.3. Aprendizagem - Suporte & $\mathrm{X}$ & & $\mathrm{X}$ & & & \\
\hline & 2.4 Deveres & & & & & & $\mathrm{X}$ \\
\hline & 2.5 Representação & $\mathrm{X}$ & & & & & \\
\hline & 2.6 Requisitos de admissão & & & & $\mathrm{X}$ & & \\
\hline \multicolumn{8}{|c|}{ 3. Avaliação } \\
\hline & 3.1 Aprendizagem formal & $\mathrm{X}$ & & & $\mathrm{X}$ & $\bar{X}$ & $\mathrm{X}$ \\
\hline & 3.2 Potenciais projectos & & & & $\mathrm{X}$ & & \\
\hline & 3.3 Cursos & & & & $\mathrm{X}$ & & \\
\hline & 3.4 Feedback dos alunos & $\mathrm{X}$ & & & & & \\
\hline
\end{tabular}

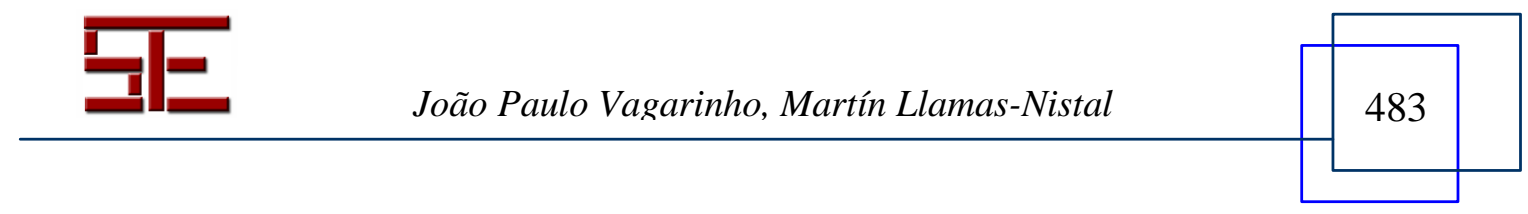


Teoría de la Educación

Educación y Cultura en la

VNiVERSIDAD

BSALAMANCA

Sociedad de la Información

TESI, 14(2), 2013, pp. 473-489

\begin{tabular}{|c|c|c|c|c|c|c|}
\hline 3.5 Organização & & $\mathrm{X}$ & & & & \\
\hline 3.6 Professores & & & & & $\mathrm{X}$ & \\
\hline \multicolumn{7}{|l|}{ 4. Cultura de qualidade } \\
\hline 4.1 Melhoria continua & & $\mathrm{X}$ & $\mathrm{X}$ & $\mathrm{X}$ & & $\mathrm{X}$ \\
\hline 4.2 Revisão periódica & & $\mathrm{X}$ & & & & \\
\hline \multicolumn{7}{|l|}{ 5. Curso } \\
\hline 5.1 Ambição & $\mathrm{X}$ & & & & & $\mathrm{X}$ \\
\hline 5.2 Aprovação & $\mathrm{X}$ & & & & $\mathrm{X}$ & \\
\hline 5.3 Créditos & & & $\mathrm{X}$ & & & \\
\hline 5.4 Desenvolvimento & & & $\mathrm{X}$ & $\mathrm{X}$ & $\mathrm{X}$ & \\
\hline 5.5 Eficácia & & & & $\mathrm{X}$ & & \\
\hline 5.6 Estrutura & & & $\mathrm{X}$ & $\mathrm{X}$ & & $\mathrm{X}$ \\
\hline 5.7 Garantia qualidade & $\mathrm{X}$ & & & $\mathrm{X}$ & & \\
\hline 5.8 Gestão & $\mathrm{X}$ & & & $\mathrm{X}$ & $\mathrm{X}$ & \\
\hline 5.9 Promoção & & & & & & $\mathrm{X}$ \\
\hline 5.10 Transferências & & & & & $\mathrm{X}$ & \\
\hline 5.11 Monitorização & $\mathrm{X}$ & & & & & $\mathrm{X}$ \\
\hline \multicolumn{7}{|l|}{ 6. Ética } \\
\hline 6.1 Institucional & & & & & & $\mathrm{X}$ \\
\hline 6.2 Questões legais & & & & $\mathrm{X}$ & & \\
\hline \multicolumn{7}{|l|}{ 7. Informação } \\
\hline 7.1 Cursos & $\mathrm{X}$ & & & $\mathrm{X}$ & $\mathrm{X}$ & $\mathrm{X}$ \\
\hline 7.2 Das avaliações & & & & & & $\mathrm{X}$ \\
\hline 7.3 Declarações politicas & & & & $\mathrm{X}$ & & \\
\hline 7.4 Eventos & $\mathrm{X}$ & & & & & \\
\hline 7.5 Financeira & & $\mathrm{X}$ & & & & \\
\hline 7.6 Pagamentos & & & & $\mathrm{X}$ & & \\
\hline 7.7 Para auditores & & $\mathrm{X}$ & & & & \\
\hline 7.8 Representação alunos & $\mathrm{X}$ & & & & & \\
\hline 7.9 Segurança & & & $\mathrm{X}$ & & & \\
\hline 7.10 Sobre estrutura & & $\mathrm{X}$ & & & & \\
\hline \multicolumn{7}{|l|}{ 8. Institucional } \\
\hline 8.1 Contractos serviços & & & & $\mathrm{X}$ & & $\mathrm{X}$ \\
\hline 8.2 Estratégia & $\mathrm{X}$ & & & & & \\
\hline 8.3 Marketing & & & & $\mathrm{X}$ & & \\
\hline 8.4 Missão e visão & & & & $\mathrm{X}$ & & $\mathrm{X}$ \\
\hline
\end{tabular}

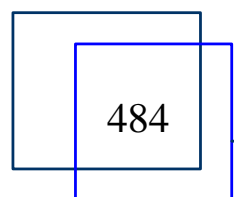




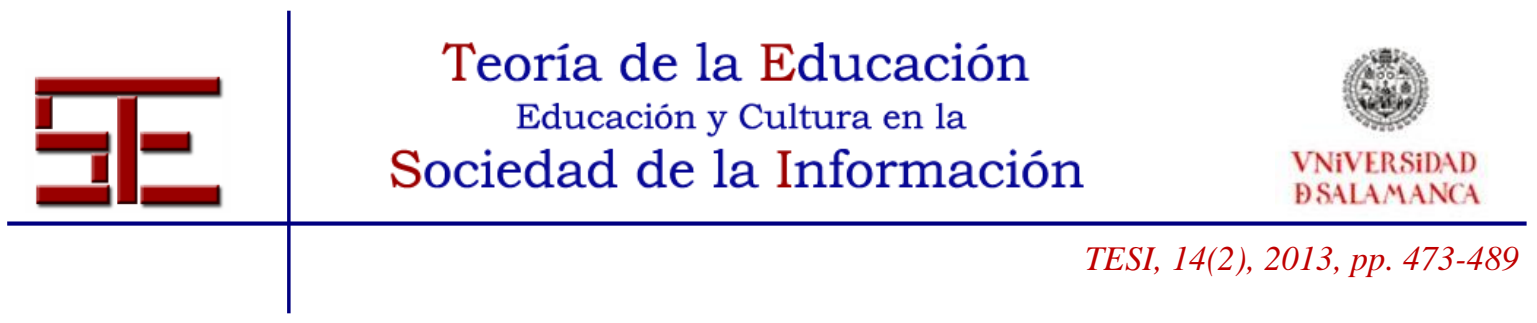

\begin{tabular}{|c|c|c|c|c|c|c|}
\hline 8.5 Objectivos & $\mathrm{X}$ & $\mathrm{X}$ & & & & \\
\hline 8,6 Políticas de transferências & & & & $\mathrm{X}$ & & \\
\hline 9. Leis & $\mathrm{X}$ & $\mathrm{X}$ & & $\mathrm{X}$ & & $\mathrm{X}$ \\
\hline 10. Materiais (Reutilização) & & & & & $\mathrm{X}$ & \\
\hline \multicolumn{7}{|l|}{ 11. Pedagogia } \\
\hline 11.1 Manual acolhimento & & & & $\mathrm{X}$ & & \\
\hline 11.2 Meio & & & & $\mathrm{X}$ & $\mathrm{X}$ & \\
\hline 11.3 Modelo pedagógico & & & & & $\mathrm{X}$ & $\mathrm{X}$ \\
\hline 11.4 Objectivos & & & & $\mathrm{X}$ & & $\mathrm{X}$ \\
\hline 11.5 Recursos Aprendizagem & & & & $\mathrm{X}$ & $\mathrm{X}$ & $\mathrm{X}$ \\
\hline 11.6 Supervisão & & & & $\mathrm{X}$ & & \\
\hline 12. Professores (Competências) & & & & $\mathrm{X}$ & $\mathrm{X}$ & \\
\hline \multicolumn{7}{|l|}{ 13. Recursos } \\
\hline 13.1 Financeiros & $\mathrm{X}$ & $\bar{X}$ & & $\mathrm{X}$ & & $\mathrm{X}$ \\
\hline 13.2 Formação & & $\mathrm{X}$ & & $\mathrm{X}$ & $\mathrm{X}$ & \\
\hline 13.3 Humanos & & & & $\mathrm{X}$ & $\mathrm{X}$ & $\mathrm{X}$ \\
\hline 13.4 Instalações & & & & $\mathrm{X}$ & & \\
\hline 13.5 De qualidade & & $\mathrm{X}$ & & & & \\
\hline \multicolumn{7}{|l|}{ 14. Segurança } \\
\hline 14.1 Segurança & & & & $\mathrm{X}$ & & $\mathrm{X}$ \\
\hline 14.2 Mecanismos controlo & & & & $\mathrm{X}$ & $\mathrm{X}$ & \\
\hline \multicolumn{7}{|l|}{ 15. Suporte } \\
\hline 15.1 Administrativo & & & & $\mathrm{X}$ & & \\
\hline 15.2 Alunos & & & & $\mathrm{X}$ & $\mathrm{X}$ & \\
\hline 15.3 Infra-estrutura & & & & $\mathrm{X}$ & & \\
\hline 15.4 Institucional & & & & $\mathrm{X}$ & & $\mathrm{X}$ \\
\hline $\begin{array}{l}\text { 15.5 Orientação, assessoria, } \\
\text { aconselhamento }\end{array}$ & & & & $\mathrm{X}$ & & $\mathrm{X}$ \\
\hline 15.6 Professores & & & $\mathrm{X}$ & $\mathrm{X}$ & & \\
\hline 15.7 Programas & & & & $\mathrm{X}$ & & \\
\hline 15.8 Serviços & & & & $\mathrm{X}$ & $\mathrm{X}$ & \\
\hline 15.9 Técnico & & & $\mathrm{X}$ & $\mathrm{X}$ & & \\
\hline
\end{tabular}

Tabela 1: Distribuição dos temas pelas entidades.

João Paulo Vagarinho, Martín Llamas-Nistal 


Teoria de la Educación
Educación y Cultura en la
$\begin{gathered}\text { VNiVERSIDAD } \\ \text { BSALAMANCA }\end{gathered}$

\section{6.- CONCLUSÃO E PROPOSTA.}

Pela análise realizada anteriormente concluímos que:

- Nenhuma entidade engloba os quinze itens referidos (Acreditação, Alunos, Avaliação, Cultura de qualidade, Curso, Ética, Informação, Institucional, Leis, Materiais, Pedagogia, Professores, Recursos, Segurança e Suporte);

- Cada item divide-se em uma ou mais categorias;

○ Quatro itens não possuem nível de detalhe (categorias) e por isso são cobertos pelas respectivas entidades que lhe fazem referência.

Podemos também concluir que todas as entidades juntas englobam todos os itens, ou seja, podemos construir um novo conjunto de dimensões. Estas dimensões são compostas como base os quinze itens apresentados anteriormente.

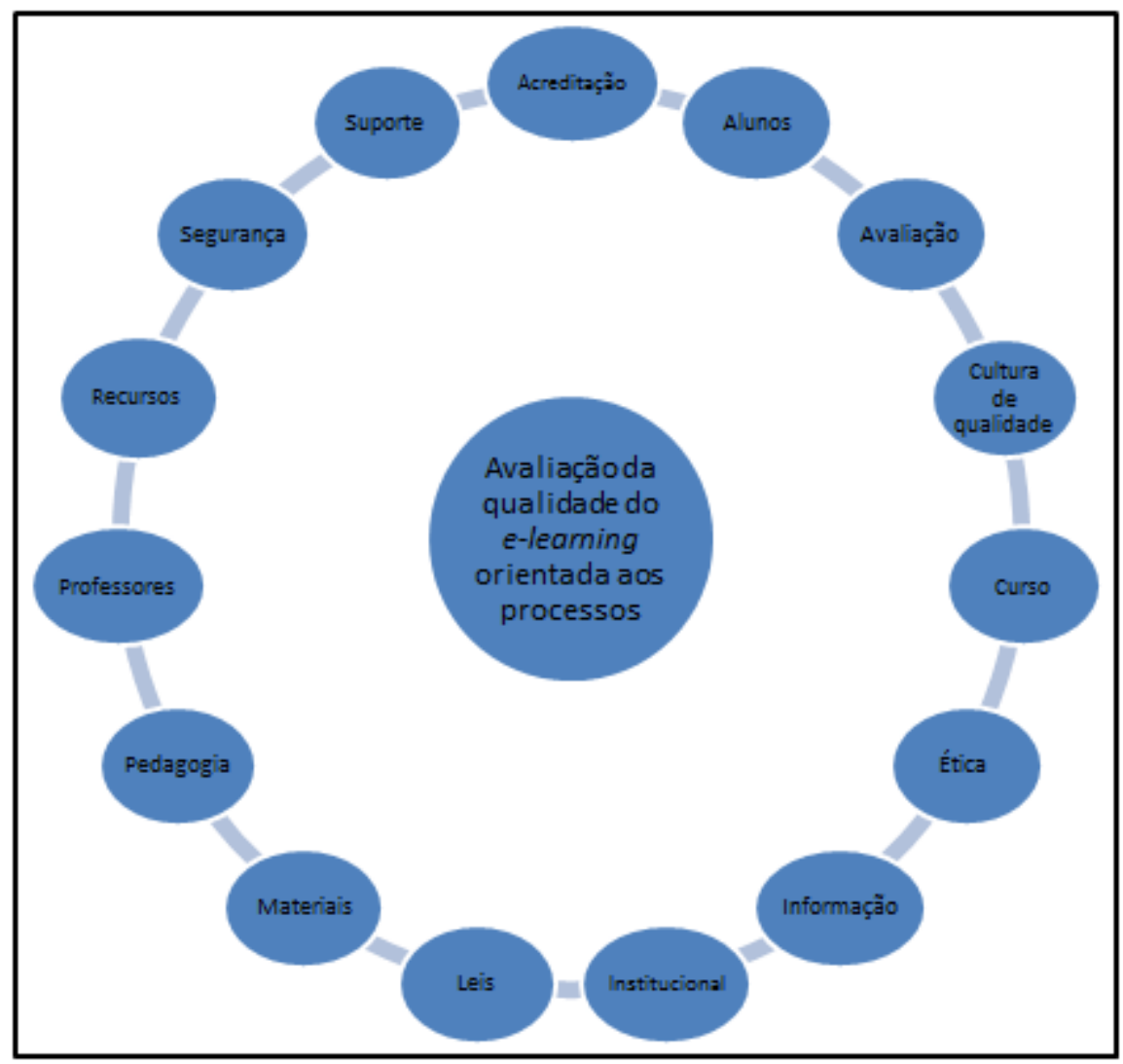

Figura 2: Dimensões da avaliação da qualidade do $e$-learning, itens principais

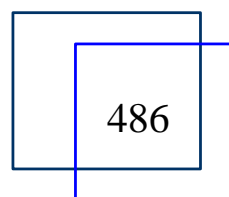

João Paulo Vagarinho, Martín Llamas-Nistal

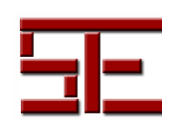




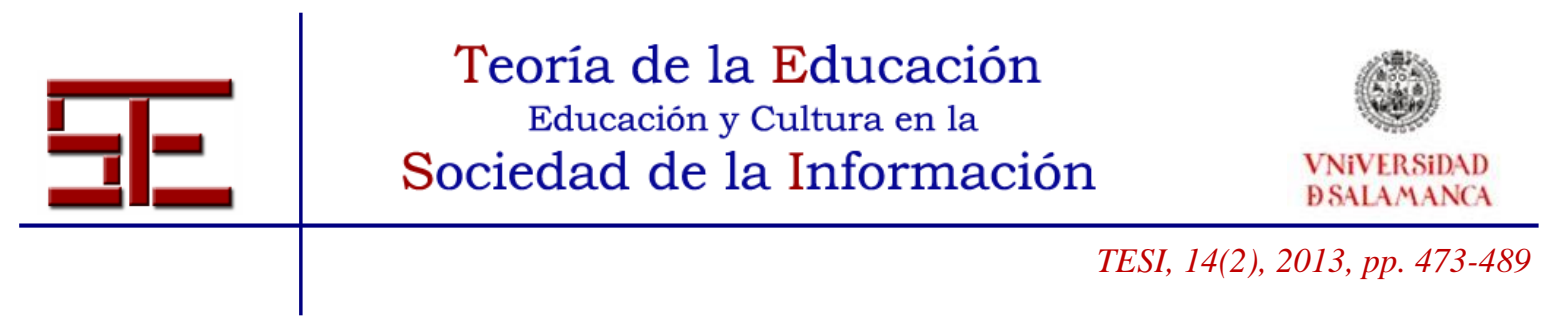

\section{8.- AGRADECIMENTOS.}

Agradecemos ao Ministério Espanhol da ciência e Inovação pelo seu apoio parcial neste trabalho sob concessão "Metodologias, Arquitecturas de padrões adaptivos e acessiveis do e-learning (Adapt2Learn)" (TIN2010-21735-C02-01).

\section{9.- BIBLIOGRAFIA.}

Meirinhos, M. \& Osório, A. (2009, Outubro). Contributos para a formação do professor x.0+1, EDUSER. Consultado em 21 de Dezembro, 2010 de https://www.eduser.ipb.pt/index.php/eduser/article/viewFile/4/3.

Cardoso, M. (2007). Comunicação informal e a emergência de comunidades de aprendizagem em contexto de E-Learning, Tese para obter titulo de mestrado, Universidade Aberta, Lisboa, Portugal.

EACEA. (2009, Julho). Ensino superior na Europa 2009: evolução ddo processo de Bolonha, Comissão Europeia. Consultado em 21 de dezembro, 2010 de http://eacea.ec.europa.eu/education/eurydice/documents/thematic_reports/099PT.pdf, consultado em Dezembro 2010.

DGES. (2010, Junho). Programa de Educação e Formação 2010. Consultado em 22 de julho, 2011 de http://www.dges.mctes.pt/DGES/pt/Reconhecimento/União+Europeia/Conselho+e+Co mité+de+Educação/Educacao+Formacao+2010.htm.

Gomes, M. (2006, Outubro). E-Learning e educação online: contributos para os princípios de Bolonha. Consultado em 10 de novembro, 2010 de http://repositorium.sdum.uminho.pt/bitstream/1822/5724/1/texto-final-bolonha.pdf.

Montalvo, E., Luna, D., Montenegro, E. (2010, Maio). La evolución y adopción de estándares en la formación virtual. Consultado em 4 de fevereiro, 2011 de http://redusoi.org/docs/publicaciones/CampoUNI1.2-LeonyUAH.pdf.

Coutiño, C. (2004, Março). Panorama general de los sistemas de educación a distancia. Consultado em 25 de março, 2011 de http://www.ateneonline.net/datos/19_01_castellanos_carlos.pdf.

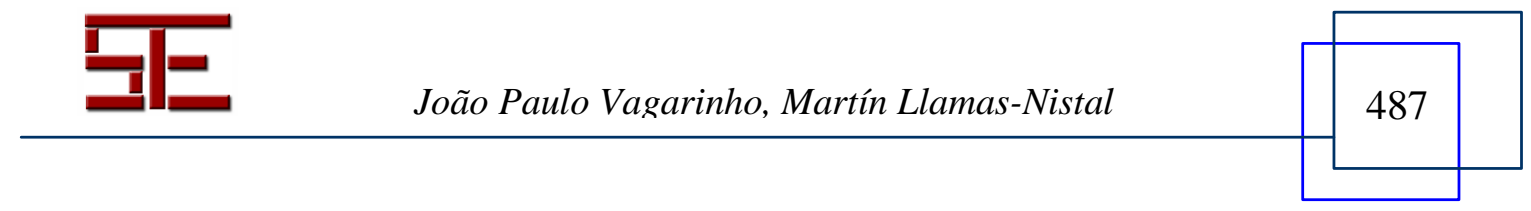




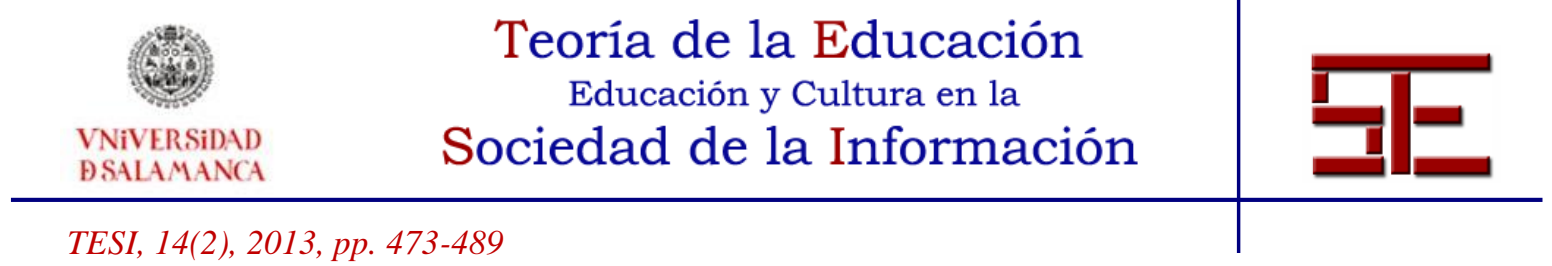

Manjón, B. et al (2007). Uso de estándares aplicados a Tic en educación. Consultado em 8 de Fevereiro, 2001 de http://ares.cnice.mec.es/informes/16/versionpdf.pdf.

Ehlers, D., Pawlowski J. (2006). Quality in European e-learning: an introduction. In Handbook on Quality and Standardisation in E-Learning. Springer, Berlin.

Dondi, C., Moretti, M. (2007, Julho). E-Learning Quality in European Universities: Different Approches for different purposes. Consultado em 2 de novembro, $2011 \mathrm{de}$ http://unique.europace.org/pdf/WP1-report-v5_FINAL.pdf.

Miranda, A., Teixeira, R. (2003, Dezembro). Em torno da distinvidade, alguns princípios fundamentais no âmbito da avaliação da qualidade de cursos online. Consultado em 16 de agosto, 2011 de http://repositorioaberto.univ-ab.pt/bitstream/10400.2/153/1/RevistaDiscursos 113-127.pdf.

Rekkedal, T. (2006, Maio).Criteria for evaluating quality in e-learning, ELQ for SME. Consultado em 2 de dezembro, 2011 de http://www.cecoa.pt/projectos/transncio/ELQ/Crit\%c3\%a9rios\%20para\%20Avalia\%c3 $\%$ a7\% c3\%a3o\%20da\%20Qualidade\%20do\%20ELearning.pdf?AspxAutoDetectCookieSupport=1.

IHEP (2000). Quality on the line. Consultado em 3 de setembro, 2011 de http://www.ihep.org/assets/files/publications/m-r/QualityOnTheLine.pdf.

WCET (2001). Best Practices For Electronically Offered Degree and Certificate Programs. Consultado em 2 de setembro, 2011 de http://wcet.wiche.edu/wcet/docs/cigs/studentauthentication/Accrediting_BestPractices.p df.

MEC/SEED (2007, Agosto). Referenciais de Qualidade para Educação Superior à Distância. Consultado em 9 de janeiro, 2012 de http://portal.mec.gov.br/seed/arquivos/pdf/legislacao/refead1.pdf.

AFT (2000). Distance Education: Guidelines for Good Practice. Washington, DC: AFT (AFL-CIO).

ODLQC (2005, Dezembro), Standards in Open and Distance Learning. Consultado em 6 de fevereiro, 2012 de http://www.odlqc.org.uk/assess-guide/standards(2005).doc.

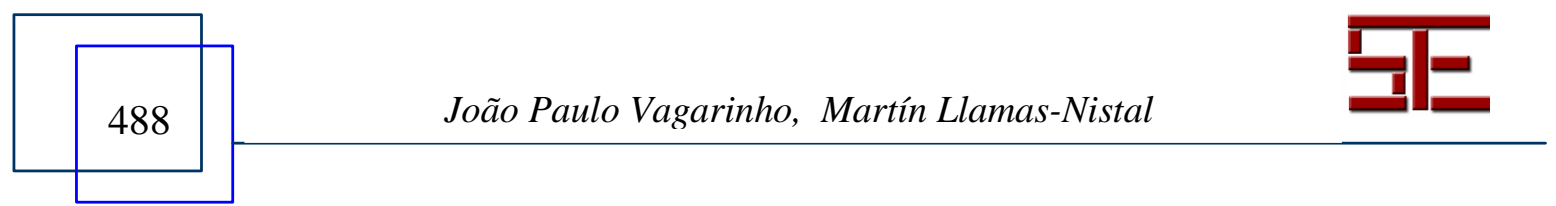




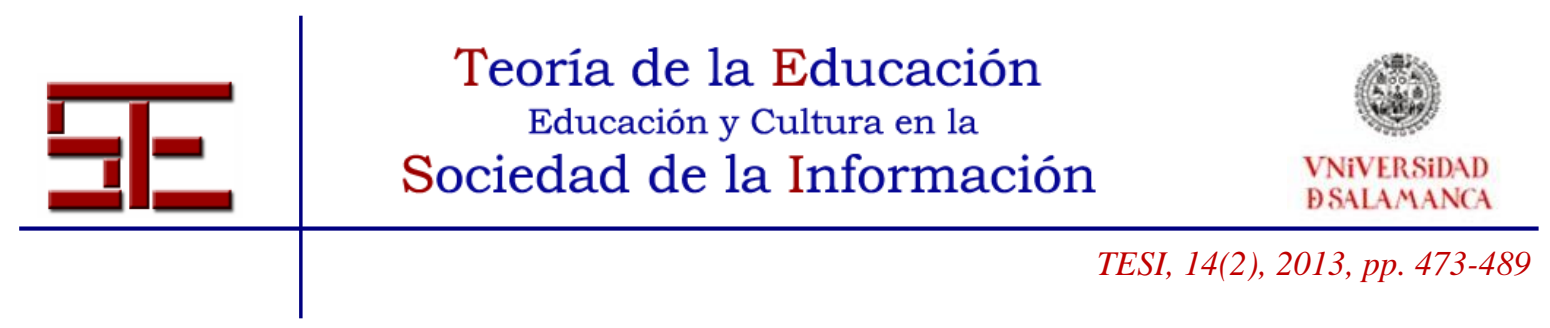

Ehlers, D. et al (2005, Outubro). Quality in e-learning. Consultado em 17 de dezembro, 2011 de http://www.etutors-portal.net/portal-contents-pt-pt/quality-and-cost-ptpt/european-quality-observatory/qualidade-em-e-learning_en.pdf/file.

Dondi, C. (2004). Quality Guide to the Non-Formal and Informal Learning Processes. $\begin{array}{llllll}\text { Consultado em } & 15 & \text { de } & \text { janeiro, } & 2012 & \text { de }\end{array}$ http://www.menon.org/publications/TQM\%20Guide\%20for\%20informal\%20learning.p df.

ELQ (2008). E-learning quality: Aspects and criteria for evaluation of e-learning in higher education. Consultado em 21 de novembro, 2011 de http://www.eadtu.nl/excellencelabel/files/0811R.pdf.

Para citar el presente artículo puede utilizar la siguiente referencia:

Vagarinho, J. P. y Llamas-Nistal, M. (2013). Avaliação na qualidade dos processos do elearning: uma proposta com novas dimensões. Revista Teoría de la Educación: Educación y Cultura en la Sociedad de la Información. 14(2), 473-489 [Fecha de consulta: dd/mm/aaaa].

http://campus.usal.es/ revistas_trabajo/index.php/revistatesi/article/view/10241/10685

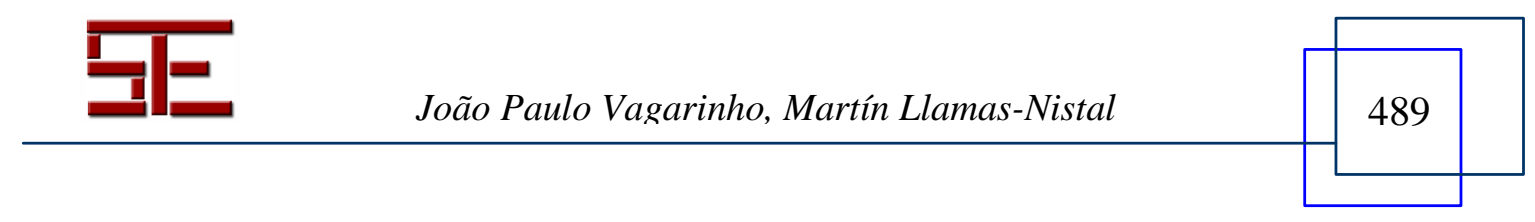

